\title{
Consideraciones técnicas para la preparación de abonos foliares de fabricación casera
}

\author{
Technical considerations for the preparation of foliar homemade fertilizers \\ Martha Orozco Aceves ${ }^{1}$ \\ José Alonso Calvo Araya ${ }^{2}$
}

Fechas de recepción: 14-3-2019

Fecha de aceptación: 14-10-2019

\begin{abstract}
Resumen
Los abonos foliares de fabricación casera constituyen una herramienta eficiente de fertilización, sobre todo en el caso de sistemas productivos manejados con enfoques agroecológicos. Algunas ventajas de estos abonos son, que su preparación no requiere equipo sofisticado, entrenamiento especializado o insumos costosos. Existe una gran variedad de abonos foliares caseros, por ejemplo, súper magro, bioles, té hidrolizado de humus, y otros; es necesario asegurarse de que es tos cumplan con la función para la cual son aplicados, que es aportar nutrientes susceptibles de ser absorbidos por vía foliar, favoreciendo la nutrición vegetal. Este es precisamente el tema a tratar en la presente revisión, específicamente, se revisan aspectos técnicos de la preparación de abonos foliares caseros a la luz de tres temas: 1) aspectos morfológicos y funcionales de la hoja, lo cual tiene incidencia directa en el proceso de absorción foliar de nutrientes, 2) la naturaleza química de los insumos (de origen vegetal, animal y mineral) utilizados en fabricación de los abonos caseros, y 3) el proceso de preparación de los mismos (hidrólisis, fermentación), lo cual posibilitará el tránsito de nutrientes a través de las barreras impuestas por la estructura foliar. Por otro lado, se mencionan mecanismos adicionales por medio de los cuales los abonos foliares caseros pueden beneficiar la producción vegetal. Por lo tanto, el objetivo de esta revisión evaluativa es analizar algunos aspectos técnicos que influirán directamente en la efectividad de los abonos foliares de fabricación casera, para predecir sus aportes al desarrollo de los cultivos.

Palabras clave: abono fermentado, abono no fermentado, cutícula, epidermis, fertilizante foliar, filoplano, nutrición vegetal.
\end{abstract}

\begin{abstract}
Homemade foliar fertilizers are an efficient fertilization tool, especially in productive systems that are managed under agroecological approaches. Some advantages of these fertilizers are that their preparation does not require sophisticated equipment, specialized training or expensive materials. There is a great variety of homemade foliar fertilizers, for example, super magro, bioles, hydrolyzed humus tea, and others; It is necessary to ensure that these fertilizers fulfill the function for which they are applied, which is to provide nutrients that can be absorbed foliarly, favoring plant nutrition. This is precisely the topic to be addressed in this review, specifically, technical aspects of the preparation of homemade foliar fertilizers are reviewed in the light of three topics: 1) morphological and functional aspects of the leaf, which has a direct impact on the process of foliar absorption of nutrients, 2) the chemical nature of the inputs (i.e., plant, animal and mineral origin) used in the manufacture of homemade fertilizers, and 3) the process of preparation (i.e., hydrolysis, fermentation), which will enable the transit of nutrients through the barriers imposed by the leaf structure. On the other hand, additional mechanisms through which homemade foliar fertilizers can benefit plant production are mentioned. Therefore, the objective of this evaluative review is to analyze some technical aspects that will directly influence the effectiveness of homemade foliar fertilizers, to predict their contributions to crop development. Keywords: cuticle, epidermis, fermented fertilizer, filoplane, foliar fertilizer, non-fermented fertilizer, plant nutrition.
\end{abstract}

1 Universidad Nacional de Costa Rica, Instituto Regional de Estudios en Sustancias Tóxicas (IRET). Ph.D. en Ecología de Suelos. Heredia, Costa Rica. Correo electrónico: martha.orozco.aceves@una.cr

2 Universidad Nacional de Costa Rica, Facultad de Ciencias de Tierra y Mar, Escuelas de Ciencias Agrarias (ECA). M.Sc. en Protección Vegetal. Heredia, Costa Rica. 


\section{Introducción}

El uso de abonos foliares de fabricación casera es una práctica que se ha vuelto popular entre los productores, sobre todo entre aquellos que practican la agricultura orgánica o ecológica. Estos enfoques de producción consideran el uso de abonos foliares de fabricación casera como una práctica amigable con el ambiente; sin embargo, es deseable verificar que estos abonos caseros estén cumpliendo la función para la cual son aplicados, que es aportar nutrientes a la planta que sean susceptibles de ser absorbidos por vía foliar. De no ser así, el productor podría estar incurriendo en una práctica que favorecería bajos rendimientos en la producción, debido a una nutrición inadecuada del cultivo y desperdiciando recursos materiales y humanos que deben invertirse en la preparación y aplicación de los abonos. En el presente trabajo se realiza una revisión de los procesos fisiológicos a nivel celular y molecular, involucrados en el proceso de absorción de nutrientes por vía foliar, también se realiza una revisión de la composición de los abonos foliares de fabricación casera. Estos aspectos aportan información de base para predecir la idoneidad y eficiencia de dichos abonos, es decir, si constituyen una fuente adecuada de nutrientes susceptibles de absorberse por vía foliar, favoreciendo la nutrición de los cultivos.

\section{Morfología y estructura de las hojas: aspectos críticos en la absorción de nutrientes por vía foliar}

La superficie aérea de la planta se caracteriza por la presencia de un complejo y diverso arreglo especializado de adaptaciones físicas y químicas que sirven para mejorar la tolerancia de las plantas a una extensa lista de factores ambientales que incluyen radiación desfavorable, temperatura, déficit de presión de vapor, viento, herbivoría, daño físico, polvo, lluvia, contaminantes, químicos de origen antropogénico, insectos y patógenos (Fernández, et al., 2013; Domínguez, et al. 2017). Estas características de las superficies aéreas de la planta que le brindan protección contra el estrés ambiental y regulan el intercambio de agua, gases y nutrientes, también constituyen los mecanismos que regulan la absorción foliar de nutrientes (Fernández and Eichert, 2009;
Fernández, et al., 2013; Domínguez, et al. 2017; Thomas, et al., 2017). La sección transversal de una hoja de una angiosperma típica consiste de una cutícula que cubre las láminas de células epidermales tanto superior como inferior (Figura 1). Estas láminas encierran el mesófilo, las hojas difieren en su estructura de acuerdo a la especie, pero generalmente consiste de parénquima en empalizada localizado junto a la epidermis superior, y parénquima esponjoso (también conocido como mesófilo esponjoso) localizado entre la lámina en empalizada y la epidermis inferior. Existen grandes espacios intercelulares entre las células del mesófilo, especialmente en el parénquima esponjoso. La epidermis es una lámina compacta que contiene en algunos casos dos o más láminas de células, y sus principales características, en relación al transporte de nutrientes y agua, son la presencia de la cutícula y de estomas (Kannan and Charnel, 1986; Salas, 2002; Fernández, et al., 2013; Domínguez, et al. 2017).

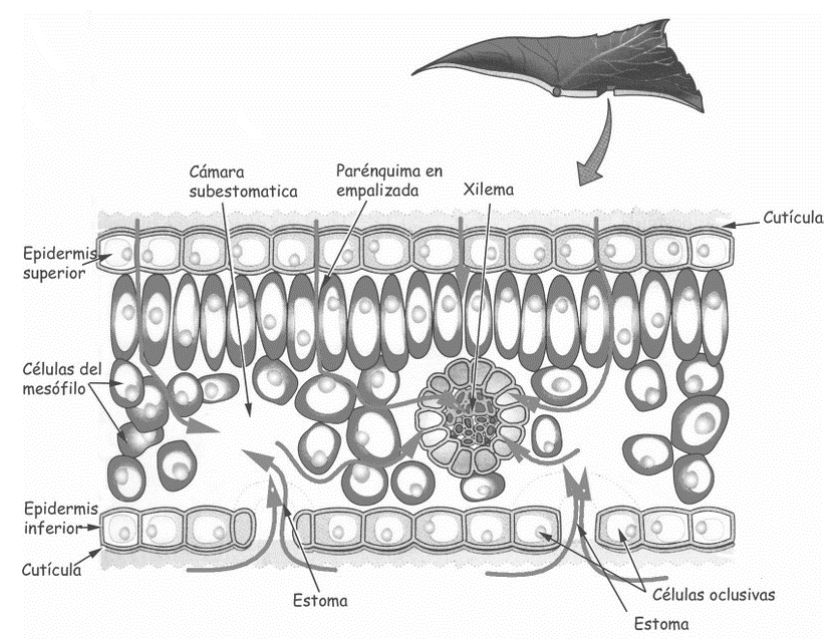

Figura 1. Sección transversal de una hoja de angiosperma. En las zonas superior e inferior se aprecia la cutícula cubriendo las láminas de células epidermales, las cuales encierran el mesófilo, que consiste de parénquima en empalizada localizado junto a la epidermis superior, y parénquima esponjoso (o mesófilo esponjoso) localizado entre la lámina en empalizada y la epidermis inferior. Las flechas ilustran las vías de penetración de abonos foliares hasta el conducto vascular. Reproducido de: Payeras (2006).

Las hojas representan la mayoría de la superficie aérea total de la planta, por lo tanto, capturan la mayoría de los nutrientes aplicados por vía foliar (Gutiérrez, 2002; Fernández, et al., 2013; Domínguez, et al., 2017). Para que los nutrientes en forma líquida, aplicados por vía foliar, 
(dentro de los cuales están incluidos los abonos foliares de fabricación casera) sean utilizados por las plantas, éstos deben seguir el siguiente proceso general de penetración: adsorción foliar, penetración cuticular, absorción en los compartimientos celulares metabólicamente activos de la hoja, translocación y utilización del nutriente absorbido por la planta (Fernández, et al., 2013; Domínguez, et al., 2017).

El requerimiento fundamental para una aplicación eficiente de nutrientes por vía foliar es que el ingrediente activo penetre la superficie de la planta de tal manera que sea metabólicamente activo en las células blanco donde el nutriente es requerido. Para esto, un abono foliar puede cruzar la superficie de la hoja a través de la cutícula per se, a través de grietas cuticulares o imperfecciones, o a través de estructuras epidermales modificadas como estomas, tricomas o lenticelas. La cutícula es una barrera efectiva contra la pérdida de agua, al mismo tiempo, es igualmente efectiva contra la absorción de sustancias químicas aplicadas foliarmente. La presencia de grietas cuticulares o la presencia de estructuras epidermales modificadas (estomas, tricomas o lenticelas) pueden contribuir significativamente a aumentar la tasa de absorción foliar de nutrientes (Fernández and Eichert, 2009; Fernández, et al., 2013; Domínguez, et al., 2017; Thomas, et al., 2017). A continuación, se describirá con mayor detalle la composición de la cutícula y de algunas estructuras epidermales.

a) Cutícula: la cutícula que cubre las superficies aéreas de las plantas es una lámina extracelular compuesta de una matriz de biopolímero que contiene ceras embebidas (ceras intracuticulares), o depositadas sobre la superficie (ceras epicuticulares) (Figura 2). En el lado interno existe una sustancia cerosa llamada cutina, la cual está mezclada con polisacáridos de la pared celular epidermal (como celulosa, hemicelulosa y pectina) en una proporción similar a la encontrada en paredes celulares vegetales. Por esta razón, la cutícula puede ser considerada como una pared celular "cutinizada". La matriz de la cutícula está comúnmente formada de un poliéster de cutina que forma redes mediante uniones éster entre grupos hidroxilo de los $\mathrm{C}_{16} \mathrm{y} \mathrm{C}_{18}$ de ácidos grasos. La composición de la matriz de biopolímero puede variar dependiendo del órgano vegetal, especie, genotipo y condiciones de crecimiento (Fernández and Eichert, 2009; Fernández, et al., 2013; Peirce, et al., 2014; Domínguez, et al., 2017).

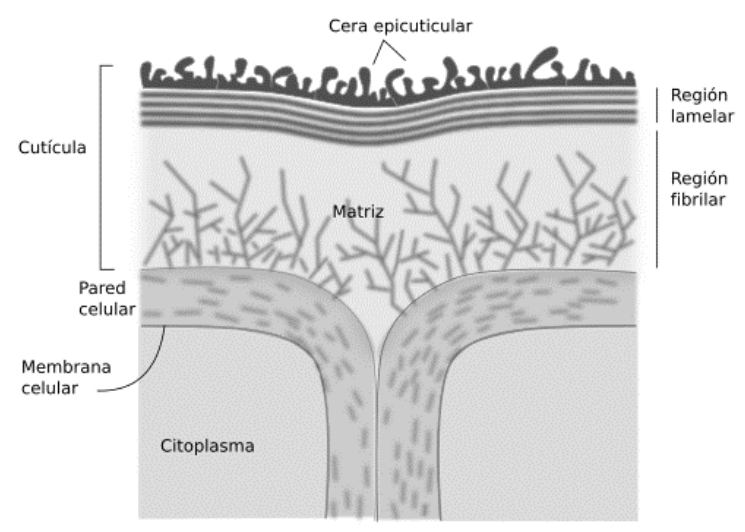

Figura 2. Detalle de una hoja mostrando la cutícula. La cutícula está formada por la lámina de cera epicuticular, que es el componente más hidrofóbico de la cutícula. Debajo de la cera epicuticular se sitúa la región lamelar, conformada por una primera lámina que contiene principalmente cutina y no presenta polisacáridos, y debajo de esta, otras láminas que consisten en cutina, pectina y hemicelulosa. La región lamelar presenta una mayor polaridad debido a la presencia de grupos funcionales hidroxilo y carboxilo. Cantidades variables de fibrillas de polisacáridos y pectina (región fibrilar) pueden extenderse desde la pared celular, uniendo la cutícula al tejido subyacente. Reproducido de: Atlas de Histología vegetal y animal. Fuente: Atlas de Histología vegetal y animal (2017).

Las ceras presentes en la cutícula, ya sea externas o embebidas en la matriz cuticular, son principalmente mezclas de moléculas alifáticas de cadena larga $\left(\mathrm{C}_{20}-\mathrm{C}_{40}\right.$ n-alcoholes, n-aldehídos, ácidos grasos de cadenas muy largas y n-alcanos) y de compuestos aromáticos (cadenas cerradas). La composición de las ceras varía de acuerdo a la especie vegetal, órgano, estado de desarrollo y las condiciones ambientales. De igual manera, cantidades variables de diferentes tipos de compuestos fenólicos pueden estar presentes en la cutícula, ya se embebidos en la matriz o químicamente unidos a la cutina o ceras mediante enlaces éster o éter (Domínguez, et al., 2017). 
b) Estructuras epidermales modificadas: entre las estructuras epidermales especializadas se encuentran los tricomas que son apéndices unicelulares o multicelulares que se originan de las células epidermales y que se desarrollan hacia afuera de la superficie de varios órganos vegetales. Los tricomas pueden crecer en todas las partes de la planta y se clasifican en glandulares o no glandulares. Mientras que los tricomas no glandulares se caracterizan por su morfología, diferentes tipos de tricomas glandulares se definen por el tipo de material que excretan, acumulan, o absorben (Gutiérrez, 2002; Segura, 2002; Fernández, et al., 2013; Domínguez, et al., 2017; Puppe and Sommer, 2018).

Los estomas son células epidermales modificadas, que controlan el intercambio gaseoso de la hoja y la pérdida transpiracional de agua. Se encuentran presentes generalmente en el lado abaxial de la hoja, pero en algunas especies, también se presentan en el lado superior de la hoja. La densidad de estomas, su morfología y funcionalidad pueden variar de acuerdo con la especie y órgano vegetal, y pueden ser afectados por factores de estrés como deficiencia de nutrientes, intensidad y calidad de luz (Segura, 2002; Fernández and Eichert, 2009; Fernández, et al., 2013; Domínguez, et al., 2017; Thomas, et al., 2017).

Las lenticelas son estructuras macroscópicas, que pueden estar presentes en la superficie de ramas, pedicelos o frutos, una vez que el peridermo se ha formado. Su origen evolutivo ha sido ligado al de los estomas, grietas epidermales y tricomas (Fernández, et al., 2013; Domínguez, et al., 2017).

\section{Vías y mecanismos de penetración a través de las hojas}

El parámetro fundamental que determina la penetración de sustancias aplicadas por vía foliar es la permeabilidad de la superficie de la hoja. Esta permeabilidad está dada por la morfología de la hoja (revisada en la sección anterior), la cual incluye diversas sustancias y estructuras que actúan como barreras al flujo de solutos (Kannan and
Charnel, 1986; Marschner, 1986; Fernández and Eichert, 2009; Fernández, et al., 2013; Peirce, et al., 2014; Pandey, 2015; Domínguez, et al., 2017; Thomas, et al., 2017). Las barreras más importantes al flujo de solutos en la hoja son:

a) Cutícula: un incremento gradual en la carga negativa desde la cera epicuticular a la lámina de pectina crea un gradiente electroquímico que puede incrementar el movimiento de cationes y moléculas de agua. Las ceras intracuticulares limitan el intercambio de agua y solutos entre la planta y el ambiente circundante, mientras que las ceras epicuticulares influyen en la humectabilidad, reflectancia a la luz y propiedades superficiales de los órganos vegetales (Kannan, 1980; Fernández and Eichert, 2009; Fernández, et al., 2013; Domínguez, et al., 2017; Thomas, et al., 2017). La naturaleza lipofílica e hidrofóbica de los componentes estructurales de la cutícula hacen de esta una barrera efectiva contra la difusión de compuestos hidrofílicos polares.

b) Pared celular: constituida de celulosa, hemicelulosa y glicoproteínas. Estos componentes constituyen una red fibrilar con poros llamados espacios interfibrilares e intermicelares, de tamaño de alrededor de $5 \mathrm{~nm}$, a través de los cuales solo pueden penetrar moléculas de cierto peso molecular (Marschner, 1986; Kannan and Charnel, 1986; Pandey, 2015). Solutos de peso molecular bajo pueden penetrar a través de estos poros mediante difusión. Moléculas muy grandes no pueden pasar a través de estos debido a su tamaño, algunas moléculas orgánicas como agentes quelantes (por ejemplo, EDTA), aminoácidos, o ácidos orgánicos son capaces de penetrar las estructuras de la hoja mediante fenómenos de difusión (Stacey, et al., 2008).

c) Membrana celular: constituye la barrera más selectiva, ya que el paso de solutos hacia el citoplasma y vacuolas está determinado por procesos más complejos como son el transporte mediado por proteínas acarreadoras específicas para iones o grupos de iones con el consiguiente 
gasto de energía en forma de ATP, o por medio de flujos electrónicos como las bombas de protones (ATPasa dependiente de protones) (Kannan, 1980; Kannan and Charnel, 1986).

\section{Tipo de moléculas susceptibles de ser absorbidas por vía foliar}

El funcionamiento de las barreras antes mencionadas en conjunto, hace que el paso de solutos a través de las células vegetales sea restringido y selectivo a moléculas de cierto peso molecular, carga electrostática y naturaleza química (Kannan, 1980; Kannan and Charnel, 1986; Marschner, 1986; Shafer and Reed, 1986; Fernández and Eichert, 2009; Pandey, 2015; Thomas, et al., 2017).

Es aceptado que la penetración de solutos a través de la cutícula constituye un proceso de difusión; sin embargo, existe evidencia del funcionamiento de una difusión facilitada en el paso de sustancias como la urea (Wen, et al., 1999; Oosterhuis, 2009; Uscola, et al., 2014). La tasa de penetración de sustancias a través de la cutícula ha sido correlacionada con el tamaño de los iones; se ha observado que moléculas orgánicas grandes como FeEDDHA (Etilendiamina di-2-hidroxifenilacetato férrico) penetran más lentamente que el Fe como $\mathrm{FeSO}_{4}$ (Kannan and Charnel, 1986; Roosta and Hamidpour, 2011; Roosta and Mohsenian, 2012).

Por otro lado, los espacios interfibrilares en las paredes celulares, a través de los cuales ocurre la absorción foliar, miden alrededor de $5 \mathrm{~nm}$, por lo tanto, únicamente moléculas de un tamaño menor a $5 \mathrm{~nm}$ serán capaces de atravesar la pared de las células epidermales en la hoja y llegar al xilema (Marschner, 1986; Kannan and Charnel, 1986; Pandey, 2015). Las dimensiones para los iones hidratados como $\mathrm{K}^{+}$y Ca ${ }^{++}$son o,66 y o,82 nm respectivamente, por lo que estos iones son capaces de atravesar la pared celular (Marschner, 1986; Kannan and Charnel, 1986; Pandey, 2015). Solutos de alto peso molecular como algunos quelatos metálicos de gran tamaño, ácidos fúlvicos, toxinas y virus son severamente restringidos por el diámetro de los poros para penetrar a través de la pared y la membrana celular (Marschner, 1986; Pandey, 2015).
En general, las características que debe tener un compuesto para ser usado en la elaboración de abonos foliares caseros son: ser muy soluble en agua y no tener efectos fitotóxicos en el follaje (Molina, 2002). Compuestos como sales minerales inorgánicas y quelatos naturales y sintéticos pueden utilizarse en la preparación de abonos foliares; sin embargo, estos tendrán capacidades variables de penetración en la hoja. Dentro de las sales minerales es posible utilizar sulfatos, nitratos y cloruros de diversos minerales, estas sales presentan buenas tasas de penetración en las hojas (Molina, 2002). Los quelatos son moléculas que se combinan con un catión metálico y lo acomplejan, formando una estructura heterocíclica de carga neutra, lo que facilita la penetración en la hoja. Los quelatos sintéticos usualmente tienen una alta estabilidad y una tasa de penetración adecuada en las hojas. Ejemplos de agentes quelatantes sintéticos son el EDTA (ácido etilendiaminotetraacético), DTPA (pentaacetato de dietilentriamina) y EDDHA (etilendiamina-N,N'-bis(ácido 2-hidroxifenilacético). Los quelatos orgánicos de cadenas cortas son agentes acomplejantes muy débiles, de poca estabilidad y baja efectividad (baja tasa de penetración en las hojas), ejemplos de éstos son los ácidos cítrico, ascórbico y tartárico. Por otro lado, los quelatos orgánicos naturales presentan diferentes grados de efectividad como agentes quelatantes, ubicándose, la mayoría de ellos, como acomplejantes intermedios con bajas tasas de penetración. Estos agentes quelatantes incluyen poliflavonoides, lignosulfatos, aminoácidos, ácidos húmicos, ácidos fúlvicos, polisacáridos, etc. (Molina, 2002).

Los ácidos húmicos y fúlvicos son especialmente importantes en los abonos foliares caseros, ya que están presentes en materiales como compost y lombricompost, los cuales se utilizan frecuentemente en la preparación de dichos abonos. Los ácidos húmicos y fúlvicos son compuestos orgánicos no muy bien definidos químicamente, son utilizados para la nutrición mineral de los cultivos debido a la acción acomplejante que ejercen sus grupos funcionales carboxílicos ( $\mathrm{COOH})$ e hidroxílicos $(\mathrm{OH})$, los cuales proveen las cargas negativas que permiten que los metales catiónicos sean acomplejados en forma de quelatos. Los ácidos húmicos y fúlvicos, también, contienen grupos funcionales amino cargados positivamente que pueden acomplejar aniones como fosfatos, sulfatos, nitratos, etc. (Molina, 2002; Singh, 2002). 


\section{Abonos foliares}

Los abonos foliares consisten en líquidos que se asperjan sobre el follaje con la finalidad de complementar la nutrición de los cultivos, así como para corregir deficiencias nutricionales de las plantas (Molina, 2002; Haytova, 2013; Li, et al., 2018). La fertilización foliar, llamada también apigea, no radicular o extra-radicular permite la aplicación de cualquiera de los nutrientes que las plantas necesitan para lograr un óptimo rendimiento. Fisiológicamente, todos los nutrientes minerales pueden ser absorbidos por vía foliar, con mayor o menor velocidad, de tal modo que teóricamente la nutrición completa de una planta podría ser satisfecha por vía foliar. Esto en la práctica no es posible, por el alto costo del elevado número de aplicaciones que sería necesario realizar para satisfacer el total de los requerimientos nutricionales de los cultivos (Molina, 2002).

El propósito de la nutrición foliar no es el de reemplazar la fertilización del suelo, ya que el abastecimiento de los principales nutrientes requeridos como en $\mathrm{N}, \mathrm{P}$ y K es más efectivo y económico a través de la fertilización del suelo. Sin embargo, la aplicación foliar ha demostrado ser un excelente método para abastecer los requerimientos de los nutrientes secundarios como Ca, Mg y S, y los micronutrientes ( $\mathrm{Zn}, \mathrm{Fe}, \mathrm{Cu}, \mathrm{Mn}, \mathrm{Bo}$ y Mo), mientras se suplemente los requerimientos de N, P y K en los periodos de crecimiento críticos del cultivo. La nutrición foliar es útil durante los estados de crecimiento, cuando disminuye la velocidad de fotosíntesis y ocurre una baja absorción de nutrientes vía raíces, en función de ayudar a la translocación de nutrientes hacia la semilla, fruto, tubérculo o crecimiento vegetativo (Gutiérrez, 2002; Haytova, 2013; Puppe and Sommer, 2018; Li, et al., 2018). Por otro lado, la nutrición foliar es una efectiva herramienta de manejo, ya que favorece e influencia los estados de crecimiento pre-productivos, compensando el estrés producido por el ambiente en condiciones adversas de crecimiento y/o una pobre disponibilidad de nutrientes (Haytova, 2013). Las aplicaciones foliares de soluciones de nutrientes se utilizan especialmente cuando (Molina, 2002; Salas, 2002; Segura, 2002; Haytova, 2013):

1. La toma de elementos desde el suelo se encuentra limitada. Su disponibilidad en el suelo está afectada por factores como el pH, contenido total, nivel y calidad de la materia orgánica, actividad de los microorganismos, antagonismos entre nutrientes presentes, etc.

2. Durante ciertas etapas críticas del desarrollo vegetal, las demandas metabólicas de nutrientes minerales pueden exceder temporalmente la capacidad de absorción de las raíces y la posterior translocación para suplir las necesidades de la planta. Esto es especialmente cierto en los cultivos de crecimiento rápido. Como consecuencia de ello, las adiciones de nutrientes al suelo no incrementan de forma apreciable la disponibilidad de estos iones para la planta, siendo necesaria otra vía que la sustituye o complemente.

3. El suministro de nutrientes vía radicular suele conllevar, a veces, grandes dosis de fertilizantes a aplicar, con los consiguientes efectos de contaminación derivados.

Existen muchos factores que influyen en la eficacia de las aplicaciones de abonos foliares. Básicamente todos ellos están relacionados con las características de la especie vegetal, las condiciones medioambientales y la disolución empleada (Cuadro 1) (Salas, 2002; Segura, 2002; Kannan and Charnel, 2008; Fernández and Eichert, 2009; Thomas et al., 2017). Existe un gran número de experiencias exitosas donde el uso de fertilizantes foliares comerciales ha logrado incrementar los rendimientos agrícolas. La fertilización foliar ha sido utilizadas en una gran variedad de cultivos de diversos tipos en muchos países del mundo, dichos cultivos incluyen: chile (Roosta and Mohsenian, 2012), caqui (Hossain and Ryu, 2009), algodón (Oosterhuis, 2009), tomate (Roosta and Hamidpour, 2011; Wen, et al., 1999), trigo (Peirce, et al. 2014), frutales (Murtic et al., 2012), maíz (Anes, et al., 2016) ginseng (Zhang, et al., 2013), con resultados positivos. La aplicación foliar de urea en algodón resultó ser absorbida eficientemente y translocada al fruto en desarrollo más cercano al punto de absorción (Oosterhuis, 2009; Sheng, et al., 2019). En olivo, la aplicación de macroelementos por vía foliar incrementó la producción de aceituna por dos años consecutivos; sin embargo, los autores establecen que la fertilización 
foliar debe complementar la fertilización edáfica y no sustituirla (Toscano, et al., 2002). La urea aplicada en invierno, incrementó el nivel foliar de $\mathrm{N}$ en granadilla morada en Australia (Menzel et al., 1986). La aplicación de nutrientes primarios como P y K y secundarios como Zn, Mn y B, incrementó la producción y calidad del fruto en cultivos como naranja dulce, maní y tomate (Tariq et al., 2007; Hossain and Ryu, 2009; Cole, et al., 2016). En maíz, la adición de N, P, K y S foliar incrementó tanto el ensilaje como el rendimiento del grano (Suwanarit and Sestapukdee, 1989; Maqsood, et al., 2017; Tejada, et al., 2018).

\section{Cuadro 1. Factores que influyen directamente en la eficacia de las fertilizaciones foliares}

\begin{tabular}{|l|l|l|}
\hline Planta & Condiciones medioambientales & Disolución \\
\hline Cera cuticular & Temperatura & Concentración \\
\hline Cera epicuticular & Luz & Cantidad aplicada \\
\hline Edad de la hoja & Fotoperiodo & Tecnología de aplicación \\
\hline Estomas & Viento & pH \\
\hline Células guarda & Humedad & Polaridad \\
\hline Tricomas & Sequía & Higroscopicidad \\
\hline Haz de las hojas & Hora del día & Tipo de compuesto \\
\hline Envés de las hojas & Potencial osmótico de la zona radicular & Estabilidad \\
\hline Turgencia foliar & Estrés nutritivo & Relaciones entre nutrientes (antagonismo y sinergismo) \\
\hline Variedad del cultivo & & Surfactantes \\
\hline Etapa del cultivo & & Calidad del agua \\
\hline Capacidad de intercambio & & Humectantes \\
\hline Estado nutricional de la planta & & \\
\hline
\end{tabular}

Adaptado de Segura (2002).

\subsection{Tipos de abonos foliares}

Los abonos foliares pueden ser: 1) preparados comerciales o 2) de fabricación casera. Una marcada diferencia entre los abonos foliares comerciales y caseros es su composición, la cual deriva de los materiales a partir de los cuales son elaborados. Los abonos foliares comerciales consisten de sales inorgánicas con cierto grado de pureza, se encuentran en muy diversas presentaciones y pueden contener tanto macro (N, P, K) (Shafer and Reed, 1986; Oosterhuis, 2009; Hossain and Ryu, 2009) como micronutrientes (Fe, $\mathrm{Zn}, \mathrm{Mn}, \mathrm{Cu}, \mathrm{Mo}, \mathrm{S}, \mathrm{B}, \mathrm{Ca}$, Mg) (Roosta and Hamidpour, 2011; Zhang et al., 2013).
Además, algunos están suplementados con fitohormonas (ácido giberélico, ácido abscísico) y vitaminas (vitamina B1) (Kannan, 2010). Para conseguir una buena cobertura de la superficie vegetal contienen además un surfactante no iónico. Algunos contienen los nutrientes en forma de quelatos, lo que permite tener a los elementos en forma soluble no ionizada, para evitar la formación de otros compuestos no asimilables por las plantas. Sin embargo, los quelatos se absorben menos eficientemente (Roosta and Mohsenian, 2012), pero una vez dentro de la planta su translocación es bastante rápida (Ferrandon and Chamel, 1988). 
Los abonos foliares caseros se elaboran a partir de materiales de la finca, estos materiales pueden ser de origen vegetal, animal o mineral (Cuadro 2) (Manual Agropecuario, 2002). Existe una gran variedad de recetas para preparar abonos foliares, por ejemplo, biofermentos, tés de estiércol, purines de hierbas, bioles, fermentos de purines, bioabonos, caldos fortificados con elementos minerales, caldos microbianos, entre otros. Los abonos de fabricación casera pueden ser sometidos a un proceso químico (por ejemplo, hidrólisis con hidróxido de potasio), o microbiano (mediante una fermentación en condiciones de anaerobiosis), previo a su uso o aplicarse inmediatamente después de ser preparados. Ejemplos específicos de abonos foliares son: fermentado de abono vacuno, supermagro, biofertilizante preparado a base de ceniza de bagazo de caña de azúcar, caldo súper cuatro, bioabono de estiércol vacuno, caldo primavesi, caldo Piamonte, caldo microbiano de rizósfera, caldo de lombricompost y caldo agromil (Manual Agropecuario, 2002; Restrepo y Hensel, 2015).

\section{Cuadro 2. Ejemplos de materiales utilizados en la elaboración de abonos foliares caseros}

\begin{tabular}{|l|l|l|}
\hline Materiales de origen animal & Materiales de origen vegetal & $\begin{array}{l}\text { Materiales de origen } \\
\text { mineral }\end{array}$ \\
\hline Estiércol de bovinos, caprinos, ovinos & Chachafruto o Balú (Eritrina edulis) & Cal dolomítica \\
\hline Leche o suero & Nacedero (Trichantera gigantea) & Bórax \\
\hline Harina de huesos & Ortiga (Urtica dioica) & Sulfato de zinc \\
\hline Vísceras & Melaza o jugo de caña & Sulfato de cobre \\
\hline Cáscaras de huevo & Plantas verdes picadas & Sulfato de magnesio \\
\hline Gallinaza & & Sulfato de hierro \\
\hline Lombricompost & & Sulfato de manganeso \\
\hline
\end{tabular}

Compilado de Molina (2002); Singh (2002); Saborío (2002).

\subsection{Efectividad de los abonos foliares de fabricación casera}

La composición química de los materiales utilizados en la fabricación de los abonos foliares caseros será un aspecto crítico en la efectividad de los mismos, es decir, determinará si estos abonos logran atravesar las barreras al paso de solutos en las hojas y complementar o corregir deficiencias nutricionales de los cultivos. La mayoría de los materiales utilizados en la elaboración de abonos foliares caseros contienen nutrientes mayores y menores en cantidades considerables (Cuadro 3) (Restrepo y Hensel, 2015); sin embargo, es necesario tomar en cuenta algunas consideraciones a la hora de preparar y aplicar dichos fertilizantes. Es importante verificar que en los abonos foliares de fabricación casera los nutrientes se encuentren en formas disponibles para ser absorbidos por las plantas por vía foliar. En el caso de abonos que contienen sales minerales (por ejemplo, súper magro, biofertilizantes, caldos minerales) (Restrepo y Hensel, 2015), los nutrientes deberían ser eficientemente absorbidos por vía foliar en forma de iones hidratados, contribuyendo a la nutrición vegetal. 
Cuadro 3. Composición química de algunos materiales utilizados en la fabricación de abonos foliares caseros

\begin{tabular}{|c|c|c|c|c|c|c|c|c|}
\hline \multirow[t]{2}{*}{ Fuentes } & \multicolumn{8}{|c|}{ Porcentajes } \\
\hline & $\mathrm{N}$ & $\mathrm{P}$ & $\mathrm{K}$ & $\mathrm{Ca}$ & $\mathrm{Mg}$ & S & $\mathrm{Fe}$ & Materia Orgánica \\
\hline Estiércol de pollera & 3,9 & 0,35 & 3,2 & 0,2 & 0,2 & & & \\
\hline Estiércol de porcinos & 0,6 & $0,3-0,5$ & $0,3-0,4$ & 0,05 & & & & 12 \\
\hline Estiércol de bovinos & $0,3-0,6$ & $0,06-0,17$ & $0,1-0,7$ & 0,1 & & & & 14 \\
\hline Estiércol de equinos & 0,4 & 0,1 & 0,8 & & & & & 21 \\
\hline Estiércol de ovinos & 0,6 & 0,3 & 0,15 & & & & & 31 \\
\hline Estiércol de chivos & 2,0 & 0,04 & 2,5 & 9,6 & 3,4 & & & \\
\hline Cachaza fresca & $0,76-0,88$ & $0,46-1,2$ & $0,29-0,65$ & $2,6-5,3$ & $0,94^{-1,88}$ & 0,25 & $0,26-0,73$ & 63 \\
\hline Bagacillo & 0,76 & 0,05 & 0,44 & 0,44 & 0,33 & 0,08 & & \\
\hline Vinaza & 0,37 & 0,05 & $2,0-4,0$ & & & & & \\
\hline Cogollo caña de azúcar & 1,0 & 0,04 & 1,05 & 0,89 & 0,26 & 0,61 & & \\
\hline Matarratón (Gliricidia sepium) & 3,73 & 0,19 & 1,94 & 2,06 & 0,6 & 0,5 & & \\
\hline Haba de burro (Canavalia ensiformis) & 2,75 & 1,49 & 1,55 & 3,92 & 2,69 & 0,54 & & \\
\hline Paja de leguminosas & $0,5^{-0,83}$ & $0,13-0,17$ & $1,7-2,0$ & $0,36-0,72$ & & & & \\
\hline Paja de cereales & $0,4-0,85$ & $0,08-0,13$ & $0,3-0,7$ & 0,24 & & & & \\
\hline Compost de basura & $0,17-0,21$ & $0,09-0,22$ & $0,54^{-0,72}$ & $7,7^{-15}, 7$ & 1,1 & & & $22-23$ \\
\hline Cenizas de leña & & & & 20,45 & & & & \\
\hline Lodos cerveceros & & 0,011 & 0,018 & 0,08 & & & & \\
\hline Amoniaco anhidro & 82 & & & & & & & \\
\hline Tiosulfato de amonio & 21 & & & & & & & \\
\hline Urea fosfato líquido puro & 9 & 22 & & & & & & \\
\hline Urea fosfato líquido & 22 & 14 & & & & & & \\
\hline Agua amoniacal & $20-25$ & & & & & & & \\
\hline
\end{tabular}

En el caso de los fertilizantes elaborados a base de compuestos orgánicos como estiércoles, lombricompost, compost, o residuos vegetales, existe la probabilidad de que los nutrientes minerales se encuentren formando moléculas complejas, de alto peso molecular, con cargas electrostáticas que no favorecen su absorción, o formando polímeros, los cuales no son capaces de atravesar las estructuras vegetales de la hoja, por lo tanto, la disponibilidad de los minerales para la nutrición vegetal podría estar limitada. Esto es especialmente importante en el caso de fertilizantes foliares, cuya preparación no incluye un proceso de fermentación o hidrolizado, (por ejemplo, tés de compost o lombricompost), y son aplicados poco después de ser preparados. En este tipo de fertilizantes, las moléculas orgánicas complejas que pueden contener nutrientes minerales, no se someten a un proceso de degradación microbiana o química, el cual contribuye a su descomposición en moléculas más 
sencillas. Como consecuencia, no se favorece la liberación de moléculas que puedan penetrar eficientemente a las plantas por vía foliar.

\section{a) Abonos foliares caseros no fermentados}

Muchos materiales utilizados para la fabricación de abonos foliares de fabricación casera contienen polímeros de moléculas orgánicas, sobre todo polisacáridos (celulosa, hemicelulosa), los cuales no son de utilidad para las plantas, ya que estas tienen la capacidad de sintetizar los carbohidratos que necesitan vía fotosíntesis. Otros materiales contienen moléculas de alto peso molecular, por ejemplo, ácidos húmicos, fúlvicos y huminas, los cuales no tienen un efecto directo en la planta, ya que estas moléculas no forman parte de los requerimientos nutricionales de las mismas. Sin embargo, los ácidos húmicos y fúlvicos pueden funcionar como quelatantes débiles de nutrientes, aspecto que fue discutido anteriormente (Singh, 2002; Saborío, 2002). No obstante, para poner a disposición de la planta los minerales acomplejados en los ácidos húmicos y fúlvicos es recomendable someter estos materiales a un proceso químico o biológico de degradación (procesos de hidrólisis y fermentación respectivamente). Los abonos foliares caseros generalmente se preparan en estañones, la hidrólisis química, realizada generalmente con hidróxido de potasio toma de 3 a 4 días, mientras que la fermentación puede variar de 20 hasta 45 días (Restrepo y Hensel, 2015).

Por lo tanto, es posible predecir que los abonos foliares caseros elaborados con materiales orgánicos, cuya preparación no incluye un proceso de degradación química o biológica aportan nutrientes susceptibles de absorberse por vía foliar de manera limitada.

A pesar de lo anterior, estos abonos tienen efectos positivos en el crecimiento vegetal, ya que actúan como permeabilizadores de la membrana celular vegetal (facilitando la absorción de nutrientes) y como promotores de crecimiento celular, debido a que contienen sustancias promotoras del crecimiento, similares a auxinas, citoquininas, ácido giberélico y ácido abscísico (Singh, 2002; Saborío, 2002; Tadayyon, et al., 2018).
Otro mecanismo (diferente al aporte directo de nutrientes), por el cual los abonos foliares de fabricación casera no fermentados pueden incrementar la producción vegetal, es promoviendo la colonización del filoplano por microorganismos benéficos. Sustancias orgánicas como ácidos húmicos, fúlvicos y otros ácidos orgánicos asperjados al follaje proveen fuentes de carbono para microorganismos saprófitos, que constituyen una barrera mecánica a la entrada de patógenos, además se proporcionan organismos antagonistas propiamente (Saborío, 2002; Zamora, et al., 2017; Tadayyon, et al., 2018;). Por lo tanto, los fertilizantes foliares caseros no fermentados pueden promover la protección natural de la planta, lo que se traduce en mayor producción de biomasa (Abbasi, et al., 2003; Litterick, et al., 2004; Liu, et al., 2016; Zamora, et al., 2017; Gwinn, 2018; Urra, et al., 2019).

Por otro lado, si los abonos foliares caseros no contienen alguna sustancia adherente o agente surfactante, es muy probable que una buena parte del líquido que es asperjado al follaje caiga al suelo. En este caso, si dichos abonos contienen materiales orgánicos, ricos en ácidos húmicos y fúlvicos, no sometidos a un proceso de fermentación, es posible predecir que tales abonos tendrán efectos positivos a nivel de suelo (y en la planta consecuentemente), por ejemplo, pueden actuar como activadores de microorganismos benéficos, aumentando la mineralización de la materia orgánica y la liberación de nutrientes, pero en este caso absorbidos a través de la raíz. Adicionalmente, los ácidos húmicos y fúlvicos incrementan la capacidad de intercambio catiónico del suelo, mejoran la estructura y la retención de humedad de este, entre otros beneficios (Lavelle and Spain, 2003; Coleman, et al., 2017).

\section{b) Abonos foliares caseros fermentados}

Cuando los fertilizantes foliares caseros se someten a un proceso de hidrólisis química (por ejemplo, con hidróxido de potasio) o degradación biológica (por microorganismos), se incrementa la posibilidad de que los minerales acomplejados en la materia orgánica (en compost, lombricompost, otros materiales vegetales) queden en formas químicas susceptibles de penetrar eficientemente por vía foliar. Algunos ejemplos de abonos foliares 
caseros fermentados son súper magro, biofertilizante preparado a base de hierbas nativas y estiércol de vaca y té de humus hidrolizado. A continuación, se realizarán algunos comentarios breves sobres estos ejemplos de abonos foliares caseros fermentados.

\section{c) Súper magro}

Los ingredientes del abono súper magro son los siguientes: estiércol de vaca, melaza, leche o suero, roca fosfatada, ceniza, sulfatos de zinc, magnesio, manganeso, ferroso y de cobre, cloruros de calcio y cobalto, molibdato de sodio y bórax. El proceso de preparación de este abono foliar consiste en una fermentación anaerobia que se extiende por aproximadamente 40 días (Restrepo y Hensel, 2015). Se trata de un abono foliar casero muy completo, ya que contiene sales minerales (sulfatos, cloruros, cenizas, roca fosfatada, bórax) de valor nutricional para las plantas que son efectivamente absorbidas por vía foliar. El estiércol de vaca proporciona microorganismos para efectuar la fermentación, también aporta minerales para la nutrición vegetal que se ponen a disposición de la planta gracias al proceso de degradación microbiana al que se someten los materiales. Además, al ser rico en materia orgánica, aporta una gran variedad de beneficios al sistema planta-suelo. La melaza y la leche (o suero) aportan carbohidratos y específicamente la leche aporta aminoácidos. Ambas sustancias constituyen una fuente de carbono y energía para los microorganismos que llevan a cabo la fermentación y que eventualmente enriquecen la microbiota del filoplano y del suelo. La leche, además, aporta aminoácidos que son útiles para las plantas y que son susceptibles de absorberse por vía foliar. En conclusión, es posible establecer que el súper magro, constituye un abono foliar casero completo, rico desde el punto de vista nutricional, y el cual es altamente probable que contenga nutrientes en formas disponibles para absorberse efectivamente por las plantas.

d) Biofertilizante preparado a base de hierbas nativas y estiércol de vaca

Los ingredientes de este abono foliar casero son: estiércol de vaca, melaza (o jugo de caña), leche (o suero), cenizas de leña o rastrojo, hierbas nativas. El proceso de preparación de este abono foliar consiste en una fermentación anaerobia que se extiende por un periodo de 20 a 30 días (Restrepo y Hensel, 2015). En el caso de este abono, las cenizas aportan nutrientes para las plantas que son efectivamente absorbidos por vía foliar. El estiércol de vaca proporciona microorganismos para efectuar la fermentación, minerales para la nutrición vegetal que hacen disponibles, por medio de la degradación microbiana, la mejora las propiedades físicas, químicas y biológicas del suelo y del filoplano. La melaza y la leche proporcionan carbohidratos que son fuente de carbono y energía para los microorganismos que realizan la fermentación y la leche aporta aminoácidos que pueden absorberse efectivamente por vía foliar. Las hierbas nativas aportan un sinnúmero de sustancias químicas, y algunas pueden actuar como agentes alelopáticos. En este caso, las cenizas pueden aportar minerales susceptibles de absorberse por vía foliar; por otro lado, el resto de ingredientes pueden mejorar la nutrición vegetal indirectamente por medio del enriquecimiento de microorganismos benéficos en el filoplano y suelo.

\section{e) Té de humus hidrolizado}

Los ingredientes de este abono foliar casero son: humus e hidróxido de potasio. El proceso de preparación de este abono foliar consiste en una hidrólisis química del humus con hidróxido de potasio la cual toma de 3 a 4 días (Restrepo y Hensel, 2015). El humus contiene nutrientes minerales acomplejados que son liberados gracias al proceso de hidrólisis, de esta manera se incrementa la probabilidad de que los nutrientes queden en formas químicas susceptibles de penetrar por vía foliar. Además, el humus hidrolizado contiene compuesto de carbono que al depositarse en la superficie de la hoja constituyen una fuente de energía para favorecer el crecimiento de organismos saprófitos, algunos con acción antagónica a organismos patógenos (Saborío, 2002; Khan, et al., 2014; Tadayyon, et al., 2018; Zamora, et al., 2017). Por últimos, en caso de caer al suelo, el humus hidrolizado ayuda a mejorar las propiedades físicas, químicas y biológicas de este (Lavelle and Spain, 2003; Coleman, et al., 2017). 


\section{Conclusiones}

En conclusión, los abonos foliares de fabricación casera constituyen una herramienta con gran potencial para ser utilizada, sobre todo, pero no exclusivamente en sistemas productivos a pequeña-mediana escala, manejados con enfoques agroecológicos o alternativos. Sin embargo, para hacer un uso eficiente de estos abonos es necesario considerar varios aspectos, para empezar, que los insumos utilizados en la preparación, por ejemplo, sales minerales, urea, cenizas, sean susceptibles de ser absorbidos por vía foliar. Por otro lado, materiales como estiércoles, compost, lombricompost, residuos vegetales o paja deberán someterse a un proceso químico o biológico de degradación para liberar los nutrientes minerales capaces de traspasar las barreras al paso de nutrientes impuestas por la hoja. Adicionalmente, los materiales orgánicos aplicados contribuyen a la salud vegetal, promoviendo una microbiota benéfica cuando caen en el suelo. Existe una gran variedad de recetas de abonos foliares caseros, algunas formuladas bajo criterios científicos con el objetivo de complementar de manera eficiente la nutrición vegetal; otras basadas en conocimientos empíricos, cuya efectividad debe ser evaluada.

\section{Bibliografía}

Abbasi, P.A.; Cuppels, D.A. and Lazarovits, G. (2003). "Effect of foliar applications of neem oil and fish emulsion on bacterial spot and yield of tomatoes and peppers". Canadian Journal of Plant Pathology, 25: 41-48.

Anees, M.A.; Ali, A.; Shakoor, U.; Ahmed, F.; Hasnain, Z. and Hussain, A. (2016). Foliar applied potassium and zinc enhances growth and yield performance of maize under rainfed conditions. International Journal of Agriculture and Biology, 18(5): 10251032.

Atlas de Histología vegetal y animal (online). Disponible en: https://mmegias.webs.uvigo.es/inicio.html [citado 21 de septiembre de 2017]. Departamento de Biología Funcional y Ciencias de la Salud. Facultad de Biología. Universidad de Vigo. España.
Cole, J.; Smith, M.; Penn, C.; Cheary, B. and Conaghan, K. (2016). "Nitrogen, phosphorus, calcium, and magnesium applied individually or as a slow release or controlled release fertilizer increase growth and yield and affect macronutrient and micronutrient concentration and content of fieldgrown tomato plants". Scientia Horticulturae, 211: 420-430.

Coleman, D.; Callaham, M. and Crossley, D. (2017). Fundamentals of Soil Ecology 3rd edition. Amsterdam: Elsevier Academic Press, 376 p.

Domínguez, E.; Heredia-Guerrero, J. and Heredia, A. (2017). "The plant cuticle: old challenges, new perspectives". Journal of Experimental Botany, 68: 5251-5255.

Fernández, V. and Eichert, T. (2009). "Uptake of hydrophilic solutes through plant leaves: current state of knowledge and perspectives of foliar fertilization". Critical Reviews in Plant Science, 28: 36-68.

Fernández, V.; Sotiropoulos, T. and Brown, P. (2013). "Foliar fertilization: scientific principles and field practices". International Fertilizer Industry Association. France, $140 \mathrm{p}$.

Ferrandon, M. and Chamel, A.R. (1988). "Cuticular retention, foliar absorption and translocation of $\mathrm{Fe}, \mathrm{Mn}$ and $\mathrm{Zn}$ supplied in organic and inorganic form”. Journal of Plant Nutrition, 11 (3): 247-263.

Gutiérrez, M.V. (2002). "Aspectos básicos de la nutrición mineral de las plantas. Absorción foliar de sustancias útiles en la aplicación de agroquímicos al follaje”. En: Meléndez, G. y Molina, E. (eds.) Fertilización foliar: principios y aplicaciones. Centro de Investigaciones Agronómicas, Universidad de Costa Rica, San José, Costa Rica. p. $1-6$.

Gwinn, K. (2018). "Bioactive Natural Products in Plant Disease Control”. Studies in Natural Products 
Chemistry, 56: 229-246.

Haytova, D. (2013). "A Review of Foliar Fertilization of Some Vegetables Crops”. Annual Review \& Research in Biology, 3 (4): 455-465.

Hossain, M.B. and Ryu, K.S. (2009). "Effect of foliar applied phosphatic fertilizer on absorption pathways, yield and quality of sweet persimmon”. Scientia Horticulturae, 122: 626-632.

Kannan, S. (1980). "Mechanisms of foliar uptake of plant nutrients: Accomplishments and prospects". Journal of Plant Nutrition, 2 (6): 717-735.

Kannan, S. and Charnel, A. (1986). "Foliar absorption and transport of inorganic nutrients". Critical Reviews in Plant Sciences, 4: 341-375.

Kannan, S. (2010). "Foliar fertilization for sustainable crop production”. En: Lichtfouse, E. (ed.) Genetic Engineering, biofertilisation, soil quality and organic farming. Springer, p: 371-402.

Khan, M.H.; Meghvansi, M.K.; Gupta, R.; Veer, V.; Singh, L. and Kalita, M.C. (2014). "Foliar spray with vermiwash modifies the arbuscular mycorrhizal dependency and nutrient stoichiometry of bhut jolokia (Capsicum assamicum)”. PLoS ONE, 9 (3): 1-8.

Lavelle, P. and Spain, A.V. (2003). Soil Ecology. USA: Kluwer Academic Publishers, 529 p.

Li, J.; Liu, H.; Wang, H.; Luo, J.; Zhang, X.; Liu, Z.; Zhang, Y.; Zhai, Y.; Lei, Q.; Ren, T.; Li, Y. and Bashir, M. (2018). "Managing irrigation and fertilization for the sustainable cultivation of greenhouse vegetables". Agricultural Water Management, 210: $354-363$.

Litterick, A.M.; Harrier, L.; Wallace, P.; Watson, C.A. and Wood, M. (2004). "The role of uncomposted materials, composts, manures, and compost extracts in reducing pest and disease incidence and severity in sustainable temperate agricultural and horticultural crop production-A Review". Critical Reviews in Plant Sciences, 23 (6): 453479 .

Liu, L.; Sun, C.; He, X.; Liu, X.; Wu, H.; Liu, M.; Tang, C. and Zhang, Y. (2016). "The secondary compost products enhances soil suppressive capacity against bacterial wilt of tomato caused by Ralstonia solanacearum”. European Journal of Soil Biology, 75: 70-78.

Manual Agropecuario. (2002). Tecnologías orgánicas de la granja integral autosuficiente. Biblioteca del campo. Bogotá: Editorial Lexus, 1093 p.

Maqsood, M.; Chattha, M.U.; Chattha, M.B.; Khan, I.; Fayyaz, M.A.; Hassan, M.U. and Zaman, Q.U. (2017). "Influence of foliar applied potassium and deficit irrigation under different tillage systems on productivity of hybrid maize". Pakistan Journal of Science, 69: 317-322.

Marschner, H. (1986). Mineral nutrition of higher plants. Great Britain: Academic Press, 674 p.

Menzel, C.M.; Simpson, D.R. and Price, G.H. (1986). "Effects of foliar-applied nitrogen during winter on growth, nitrogen content and production of passionfruit”. Scientia Horticulturae, 28(4): 339346.

Molina, E.A. (2002). "Fuentes de fertilizantes foliares". En: Meléndez, G. y Molina, E. (eds.) Fertilización foliar: principios y aplicaciones. Centro de Investigaciones Agronómicas, Universidad de Costa Rica, San José, Costa Rica. p. 26-35.

Murtic, S.; Civic, H.; Duric, M.; Šekularac, G.; Kojovic, R.; Kulina, M. and Krsmanovic, M. (2012). "Foliar nutrition in apple production”. African Journal of Biotecnology, 11:10462-10468.

Oosterhuis, D.M. (2009). "Absorption of foliar-applied nitrogen by cotton". The Proceedings of the 
International Plant Nutrition Colloquium XVI. UC Davis.

Pandey, R. (2015). “Mineral Nutrition of Plants”. En: Bahadur B.; Venkat Rajam M.; Sahijram L. and Krishnamurthy, K. (eds). Plant Biology and Biotechnology. New Delhi: Springer, 827 p.

Payeras, A. (2006). “Abonos foliares”. Bonsái Actual No. 13 (on line). Disponible en: http://www. bonsaimenorca.com/articulos/articulos-tecnicos/ abonos-foliares/ [citado 21 de septiembre de 2017]. Bonsai Menorca, Escuela de Bonsái de Antonio Payeras.

Peirce, C.A.E.; McBeath, T.M.; Fernández, V. and McLaughlin, M.J. (2014). "Wheat leaf properties affecting the absorption and subsequent translocation of foliar-applied phosphoric acid fertiliser". Plant and Soil, 384: 37-51.

Puppe, D. and Sommer, M. (2018). "Experiments, uptake mechanisms, and functioning of silicon foliar fertilization: A review focusing on maize, rice, and wheat”. Advances in Agronomy, 152: 1-49.

Restrepo, R.J. and Hensel, J. (2015). "El A, B, C de la agricultura orgánica, fosfitos y panes de piedra”. Colombia. 397 p.

Roosta, H.R. and Hamidpour, M. (2011). "Effects of foliar application of some macro- and micro-nutrients on tomato plants in aquaponic and hydroponic systems". Scientia Horticulturae, 129: 396-402.

Roosta, H.R. and Mohsenian, Y. (2012). "Effects of foliar spray of different Fe sources on pepper (Capsicum annum L.) plants in aquaponic system”. Scientia Horticulturae, 146: 182-191.

Saborío, F. (2002). Bioestimulantes en fertilización foliar. En: Meléndez, G. y Molina, E. (eds.) Fertilización foliar: principios y aplicaciones. Centro de Investigaciones Agronómicas, Universidad de Costa Rica, San José, Costa Rica. p. 107-124.
Salas, R.E. (2002). "Herramientas de diagnóstico para definir recomendaciones de fertilización foliar". En: Meléndez, G. y Molina, E. (eds.) Fertilización foliar: principios y aplicaciones. Centro de Investigaciones Agronómicas, Universidad de Costa Rica, San José, Costa Rica. p. 7-18.

Segura, A. (2002). "Fertilización foliar: principios y aplicaciones”. En: Meléndez, G. y Molina, E. (eds.) Fertilización foliar: principios y aplicaciones. Centro de Investigaciones Agronómicas, Universidad de Costa Rica, San José, Costa Rica. p. 19-25.

Shafer, W.E. and Reed, D.W. (1986). "The foliar absorption of potassium from organic and inorganic potassium carriers". Journal of Plant Nutrition, 9: $143-157$.

Sheng, F.; Gao, K.; Hu, W.; Wang, S.; Chen, B. and Zhou, Z. (2019). "Foliar and seed application of plant growth regulators affects cotton yield by altering leaf physiology and floral bud carbohydrate accumulation”. Field Crops Research, 231: 105-114.

Singh, B.K. (2002). "Fertilización foliar de cultivos con ácidos húmicos”. En: Meléndez, G. y Molina, E. (eds.) Fertilización foliar: principios y aplicaciones. Centro de Investigaciones Agronómicas, Universidad de Costa Rica, San José, Costa Rica. p. 101-106.

Stacey, S.P.; Oosterhuis, D.M. and McLaughlin, M.J. (2008). "The effect of chelating agents on the foliar sorption of zinc fertilizers". The Americas Journal of Plant Science and Biotechnology, 2(2): 69-73.

Suwanarit, A. and Sestapukdee, M. (1989). "Stimulating effects of foliar K-fertilizer applied at the appropriate stage of development of maize: A new way to increase yield and improve quality". Plant and Soil, 120: 111-124.

Tadayyon, A.; Naeimi, M. and Pessarakli, M. (2018). "Effects of vermicompost and vermiwash 
biofertilizers on fenugreek (Trigonella foenum) plant". Communications in Soil Science \& Plant Analysis, 49 (19): 2396-2405.

Tejada, M.; Rodríguez-Morgado, B.; Gómez, I.; FrancoAndreu, L.; Benítez, C. and Parrado, J. (2016). "Use of biofertilizers obtained from sewage sludges on maize yield”. European Journal of Agronomy, 78: 13-19.

Thomas, B.; Murray, B. and Murphy, D. (2017). Encyclopedia of Applied Plant Sciences. 2nd edition. Amsterdam: Elsevier Academic Press, $1706 \mathrm{p}$.

Urra, J.; Alkorta, I.; Lanzen, A.; Mijangos, I. and Garbisu, C. (2019). "The application of fresh and composted horse and chicken manure affects soil quality, microbial composition and antibiotic resistance". Applied Soil Ecology, 135: 73-84.

Uscola, M.; Villar-Salvador, P.; Oliet, J. and Warren, C.R. (2014). "Foliar absorption and root translocation of nitrogen from different chemical forms in seedlings of two Mediterranean trees". Environmental and Experimental Botany, 104: $34-43$.

Wen, T.X.; Ikeda, H. and Oda, M. (1999). "Absorption, translocation, and assimilation of foliar applied urea compared with nitrate and ammonium in tomato plants". Soil Science and Plant Nutrition, 45 (3): 6o9-616.

Zamora, K.; Castro, L.; Wang, A.; Arauz, L.F. and Uribe, L. (2017). "Uso potencial de lixiviados y tés de vermicompost en el control del ojo de gallo del cafeto Mycena citricolor”. Agronomía Costarricense, 41: 33-51.

Zhang, H.; Yang, H.; Wang, Y.; Gao, Y. and Zhang, L. (2013). "The response of ginseng grown on farmland to foliar-applied iron, zinc, manganese and copper”. Industrial Crops and Products, 45: 388-394. 